\title{
Entre intelectuales y expertos: una aproximación a los debates norteamericanos sobre los medios de comunicación (1940-1950)
}

Emiliano Sánchez Narvarte

emiliano.sanchez@perio.unlp.edu.ar

Consejo Nacional de Investigaciones Científicas y Técnicas (CONICET) Universidad Nacional de La Plata, Instituto de Investigaciones Aníbal Ford

Recibido: 15 de marzo de 2018 Aceptado: 22 de octubre de 2018.

\section{Resumen}

Este artículo analiza los debates que se produjeron en un sector de la intelectualidad de los Estados Unidos en torno a la relación entre los medios de comunicación y la sociedad, entre mediados de la década del cuarenta y principios de la del cincuenta. Puntualmente, se analizan las reflexiones teóricas de autores como Paul Lazarsfeld, Harold Lasswell, Robert Merton, y Gladys y Kurt Lang. Se aborda el análisis a partir de la indagación de los itinerarios académicos de los autores mencionados y sus posicionamientos dentro del campo intelectual norteamericano. Desde aquí se reconstruyen tres ejes que preocuparon -de manera disímil- a este conjunto de investigadores: las discusiones sobre los procesos de democratización cultural, los límites y alcances de las funciones de los medios masivos en la sociedad y, por último, la cuestión de la influencia política de los medios. Consideramos que pensar relacionalmente las producciones de estos autores permite visualizar las divergencias y los matices de esta franja de la tradición norteamericana de la comunicación conocida como Mass Communication Research.

Palabras clave: teoría de la comunicación; medios masivos; funcionalismo norteamericano; efectos de la comunicación.

\section{Among intellectuals and experts: a look at American debates on communications media (1940-1950)}

\begin{abstract}
This article considers the discussions that part of the United States intelligentsia, in the late 1940s and early 50s, had about the relationship between communications media and society. Specifically, it delves into the theoretical reflections of authors such as Paul Lazarsfeld, Harold Lasswell, Robert Merton, and Gladys and Kurt Lang. Our analysis stems from our exploration of the academic itineraries of the aforementioned authors and their place among American
\end{abstract}


intellectuals. With this as our starting point, we reconstruct three lines of inquiry that, with varying degrees of intensity, preoccupied this group of investigators: processes of cultural democratization, the reach and limits of mass media's social function, and media's political influence. By thinking about how the outputs of these authors relate to each other, we can visualize the divergences and nuances within the American communications tradition known as Mass Communication Research.

Key words: communication theory; mass media; American functionalism; the effects of communication.

\section{Entre intelectuais e expertos: uma aproximação aos debates norte-americanos sobre os meios de comunicação (1940-1950) \\ Resumo}

Este artigo analisa os debates que ocorreram em um setor da intelligentsia dos Estados Unidos em torno à relação entre os meios de comunicação e a sociedade, entre meados dos anos quarenta e o início dos anos cinquenta. Especificamente, são analisadas as reflexões teóricas de autores como Paul Lazarsfeld, Harold Lasswell, Robert Merton e Gladys e Kurt Lang. O analise é abordado reconstruindo os itinerários acadêmicos dos autores acima mencionados e seu posicionamento no campo intelectual norte-americano. A partir daqui, são identificados três eixos que ocuparam -de forma diferente- a este grupo de pesquisadores: as discussões em torno dos processos de democratização cultural; os limites e alcances das funções dos meios de comunicação na sociedade e, finalmente, a questão da influência política dos meios de comunicação social. Consideramos que pensar concomitantemente as produções de cada um desses autores, permite visualizar as divergências e as nuances dentro dessa franja da tradição norte-americana em comunicação conhecida como Mass Communication Research.

Palavras chave: teoria da comunicação; meios de comunicação; funcionalismo norte-americano; efeitos da comunicação.

\section{Introducción}

En este artículo se indagarán las diferentes modalidades de reflexión que emergieron en torno a la cultura de masas en general y sobre los medios masivos en particular, en una franja de investigadores, profesionales e intelectuales de los Estados Unidos, entre mediados de la década del cuarenta y principios de la década del cincuenta. No nos interesa poner a prueba la cientificidad de los conceptos ni los marcos de interpretación utilizados por la comúnmente denominada Mass Communication Research (De Moragas, 2011). Sí, en cambio, pretendemos esbozar un mapa de los problemas y las posiciones que configuraron los debates alrededor de la cultura de masas y los mass media.

El investigador canadiense Denis McQuail, en su trabajo Mass communication theory, ha sostenido que el desarrollo de los nuevos medios en el devenir del siglo XX generó una serie de preocupaciones vinculadas con su gravitación tanto en la política como en la cultura: en relación con la primera, los vio como un elemento esencial de la vida democrática en cuanto arena y canal de los debates públicos, pero también, paradójicamente, fueron pensados como medios para ejercer el poder para un sector 
político relativamente privilegiado. Con respecto a la cultura, para este autor, los medios masivos se constituyeron en una fuente básica de producción de imágenes de la realidad social y fueron ocupando un lugar cada vez mayor en el tiempo de ocio de las sociedades (McQuail, 2003 [1983], p. 4).

En cuanto a las condiciones específicas de emergencia del pensamiento norteamericano sobre la comunicación, Miquel de Moragas (2011, p. 60) afirma que este no puede ser desconectado de los procesos de irrupción del fascismo y del estalinismo, y su relación con la aplicación de métodos de propaganda. Complementariamente, tampoco se deben olvidar los éxitos de audiencia obtenidos por las empresas de radio privadas. En este cruce de orientaciones diferentes, se fue difundiendo la idea de que los medios de comunicación eran "instrumentos de persuasión eficaces" cuando "incidían en receptores desprotegidos” (p. 61).

La pregunta acerca de qué influencia y qué efectos causaban los medios y cómo la sociedad tomaba decisiones fue una inquietud transversal a distintos campos, del político al empresarial, del científico y académico al militar. La investigación sobre la comunicación de masas se convirtió en un modo de responder a las necesidades de una época que se repartía entre distintas "urgencias": tanto las de establecer mejores estrategias de comunicación electoral e interpelar a la opinión pública, como también las de pensar la publicidad como factor clave de una economía postdepresión y de importancia creciente en la financiación de los medios (p.59).

En relación con lo anterior, nuestra hipótesis de trabajo es que en Estados Unidos la producción de conocimiento con respecto a comunicación se constituyó, entre finales de los años cuarenta y principios de los cincuenta, a partir de un espacio de intersecciones definido por el Estado, el mundo de la academia, el campo empresarial y lo que se denomina campo intelectual. Esto fue posible, en líneas generales, porque la inquietud sobre el rol de los medios en la sociedad interpeló a distintos sectores que vieron en los mass media oportunidades y desafíos políticos o comerciales. Para dar cuenta de ello, no analizaremos las producciones teóricas emergentes de manera diacrónica, sus desarrollos y evoluciones a lo largo del tiempo, como aparecen en importantes e ineludibles estudios que abordan la cuestión (De Moragas, 1985 y 2011; Mattelart, 1996; Mattelart \& Mattelart, 1997; McQuail, 2003 [1983]; Wolf, 2013 [1987]). Por el contrario, la indagación sincrónica nos permitirá pensar procesos y producciones que se dieron de forma paralela y, desde una perspectiva relacional, identificaremos regularidades, así como también posicionamientos divergentes.

\section{Abordaje teórico-metodológico}

Reconstruir, aun parcialmente, los itinerarios y las trayectorias de los intelectuales analizados permite dar cuenta de la heterogeneidad de instancias en las que se produjo 
el conocimiento especializado sobre lo social. En relación con esto, la noción de espacios de intersecciones múltiples, formulada por Neiburg y Plotkin (2004, p. 18), nos permitirá indicar los pasajes de algunos de los referentes por instituciones, formaciones y organizaciones culturales diversas, así como por organismos estatales y académicos. La propuesta de Neiburg y Plotkin nos resulta de utilidad en términos analíticos en tanto nos habilita a pensar que la producción de conocimiento sobre lo social aflora de la confluencia entre espacios distintos. En este sentido, estos autores proponen indagar los cruces, los "circuitos de circulación y vínculos entre la universidad, la empresa privada, los emprendimientos intelectuales y la gestión pública" (Neiburg \& Plotkin, 2004, p. 19).

De modo complementario, consideramos pertinente el planteo de Raymond Williams (2015 [1981]) con respecto a no tomar a los intelectuales o a los académicos como un sector representativo de la organización social de los productores culturales (p. 178). El sociólogo galés entiende que reducir la categoría intelectuales a ciertos tipos de escritores, filósofos y pensadores sociales excluye a otros agentes que contribuyen a la cultura general: aquellos que están situados en las instituciones políticas, económicas, sociales y religiosas; funcionarios públicos; expertos en finanzas que, dirá Williams, "están directamente implicados en la producción y reproducción del orden social y cultural general" (p. 180). El autor ubica a los intelectuales como una actividad especializada dentro de la categoría más general de productores culturales.

En lo estrictamente vinculado con el estudio de las obras, el investigador Roger Chartier propone un análisis que articule el cruce de dos líneas. Una es vertical o diacrónica, para establecer la relación de un texto o un pensamiento con expresiones previas de la misma rama del campo cultural. La otra es horizontal o sincrónica: esta lectura puede señalar correspondencias entre el producto intelectual específico y las producciones emergentes en otros campos o aspectos de una cultura al mismo tiempo (Chartier, 1992, p. 41).

En cuanto al criterio de selección de los referentes y sus respectivos textos, identificaremos el eje común sobre el cual se han fundado los debates y, a partir de esto, estableceremos cuáles fueron las posiciones y las reflexiones que surgieron. Consideramos que desde esta clave se puede echar luz sobre los matices y la diversidad que caracterizó el pensamiento sobre la comunicación de una franja de intelectuales y expertos norteamericanos.

En un contexto atravesado por la pregunta por los efectos de la masificación de la cultura, entre la supuesta decadencia y la democratización (Swingewood, 1979, p. 93), los trabajos de Harold Lasswell (1985 [1948]) y Paul Lazarsfeld y Robert Merton (1985 [1948]) permiten identificar una dimensión de las discusiones en torno a las funciones que los medios podían cumplir en la sociedad. Respecto a la propaganda política y la capacidad de los medios masivos de provocar cambios en las actitudes del 
Austral Comunicación

Volumen 7 número 2 (diciembre de 2018): 155-172. ISSN 2313-9129

electorado o en sus prácticas de consumo, recuperamos las reflexiones surgidas de las principales investigaciones de Lazarsfeld (1940), Merton (1949), y Lazarsfeld, Berelson y McPhee (1985 [1954]). El contrapunto de esos planteos lo produjeron Kurt y Gladys Lang (1985 [1955]), quienes criticaron explícitamente no solo las conclusiones, sino también la estrategia teórico-metodológica elaborada por el equipo de trabajo liderado por Lazarsfeld.

\section{Breves consideraciones históricas}

En el marco de las crisis políticas originadas por el crac de 1929, gran parte de las fuerzas gobernantes que a finales de los veinte y principios de los treinta se encontraban en el poder habían cambiado. El abismo económico, como denominó Eric Hobsbawm (2006 [1994]) a este proceso, evidenció, al menos en su momento, que el "viejo liberalismo estaba muerto o parecía condenado a desaparecer" (p. 114). En este contexto asomaron a la arena política tres matrices que lucharon por la hegemonía político-intelectual: una vinculada con el comunismo marxista, representada por la Unión Soviética; otra inscrita en un capitalismo que -en franco abandono de los principios del libre mercado- se articuló con movimientos socialdemócratas y organizaciones obreras no comunistas; en tercer lugar, el fascismo, que, tras la Gran Depresión, se convirtió en un movimiento mundial (p. 114).

La particular situación norteamericana, en líneas generales, adoptó la segunda de estas matrices. A lo largo de la década del treinta, el gobierno de Franklin D. Roosevelt (1933-1945) había concretado medidas como la Ley de Seguridad Social, la Administración para el Progreso del Empleo, la Ley Nacional de Relaciones Laborales y la Ley Nacional de Vivienda (Gómez Aguirre \& Resico, 2009, p. 42). La reconfiguración de los modos de organización de los trabajadores tuvo un impacto de relevancia debido a que, a partir de la Ley de Recuperación de la Industria Nacional (NIRA, por sus siglas en inglés), los sindicatos se vieron fortalecidos al contar con mejores condiciones para negociar con los empleadores en el marco del Consejo Nacional del Trabajo (Gómez Aguirre \& Resico, 2009).

Sobre finales de la década y de forma complementaria a lo anterior, se inició un proceso de reorientación belicista, de rearme, y una clara alineación con los países occidentales. Estos factores fueron preparando a la sociedad norteamericana para el conflicto armado que se iniciaría en 1939.

\section{Itinerarios: entre la academia, la experticia y el campo intelectual}

Harold Lasswell (1902-1978), formado en sociología, se relacionó tempranamente con diversas problematizaciones de la política, la guerra y los medios de comunicación 
masiva, temas sobre los que desarrolló su tesis doctoral en 1927. Tras un paso formativo por Inglaterra, Francia, Suiza y Alemania, retornó a los Estados Unidos. En este país fue uno de los fundadores del Institute for Propaganda Analysis (IPA) y participó de este proyecto entre 1937 y 1941. Lo relevante fue el tipo de preocupaciones que emergieron allí. Sus integrantes se propusieron realizar estudios acerca del contenido de las propagandas de los medios masivos para develar su eficacia. Con la publicación del boletín mensual del IPA, se pretendía educar a la población en la lectura crítica de los medios para "ayudar al ciudadano a analizar la propaganda" (como se cita en Wolf, 2013 [1987], p. 31).

En este sentido, como observa Graham (2015), el universo intelectual en el que se inscribió Lasswell estaba interpelado por las reconfiguraciones de la vida social y política tras la crisis de 1929, la reorientación de la vida democrática durante el gobierno de Roosevelt y el ascenso de los movimientos totalitarios europeos. Al respecto, desde el IPA asumieron un posicionamiento de "defensa de la democracia" (Graham, 2015, p. 36), en el marco de lo que entendieron como un asedio a la vida institucional norteamericana durante la Segunda Guerra Mundial.

Sin duda, la articulación entre medios, política y poder fue un eje de las investigaciones de Lasswell a lo largo de su trayectoria. Su teorización acerca de la función de los medios debe ser conectada con su posicionamiento intelectual frente al proyecto de sociedad que se estaba configurando en la década del cuarenta en Estados Unidos tras la Gran Depresión.

Por su parte, los sociólogos Gladys Lang (1919-2016) y Kurt Lang (1924) se formaron en la Escuela de Chicago y realizaron toda su producción intelectual en conjunto. Gladys fue research assistant en la United States Office of War Information (OWI), donde elaboró sus primeros trabajos en relación con los medios masivos, y Kurt colaboró como research assistant en el ejército. Ya en sus primeras investigaciones, estrechamente ligadas a las necesidades de las entidades estatales de las que formaban parte, pensaron la articulación entre medios masivos, propaganda y opinión pública. Los organismos políticos y militares en los que participaron fueron verdaderos laboratorios de prueba de políticas que apuntaban a ensayar distintas estrategias de interpelación a la sociedad, en un contexto bélico de inicio de la Guerra Fría. Sus primeros estudios, de inicios de la década del cincuenta, intentaron poner de relieve los efectos que, a corto o a largo plazo, ejercían los medios de comunicación sobre la sociedad. En este sentido, se ubicaron en las antípodas de uno de los más importantes investigadores norteamericanos del siglo XX: Paul Lazarsfeld.

Lazarsfeld (1901-1976), austríaco de nacimiento y formado en matemática, tuvo una temprana preocupación por la psicología social. Como producto de una historia familiar vinculada con la izquierda de su país, en su juventud se incorporó en los 
Austral Comunicación

Volumen 7 número 2 (diciembre de 2018): 155-172. ISSN 2313-9129

campamentos socialistas juveniles como educador de los hijos de las familias trabajadoras (Cole, 2004, p. 3). La preocupación por la situación social y económica de la clase obrera se condensó en un estudio de 1930 en coautoría con Marie Jahoda y Hans Zeisel, Marienthal: The Sociography of an Unemployed Community, en el que investigó las condiciones laborales de Marienthal, un pueblo al sur de Viena cuya población evidenciaba altos índices de desocupación (Jahoda, Lazarsfeld \& Zeisel, 2009 [1930]). Este trabajo, en el contexto de un proceso más amplio de migración intelectual europea hacia los Estados Unidos, resultado de los procesos de censura, proscripción y persecución en distintos países de Europa (Fleming \& Bernard, 1969; Jay, 2017), le dio visibilidad ante distintos referentes europeos -fundamentalmente, los parisinos- de la Fundación Rockefeller, y obtuvo una beca de estudio en los Estados Unidos en 1933.

Dos años más tarde, en Nueva Jersey, gracias a sus lazos con Hadley Cantril y Frank Stanton, empezó a organizarse el Proyecto de Investigación de Radio Princeton, conocido en el mundo intelectual como Princeton Radio Research Project (De Moragas, 2011, p. 79). Como sostienen Müller-Doohm (2005) y Supa (2009), la preocupación central residía en cómo la radio se incorporaba en la vida cotidiana de los estadounidenses, por qué la escuchaban y qué tipo de programación consumían.

Otro de los animadores intelectuales fue el sociólogo Robert King Merton (19102003). Hacia finales de la década del veinte se acercó a la sociología de la mano del exiliado soviético Pitirim Sorokin, quien fundó el Departamento de Sociología de la Universidad de Harvard, donde se formaría Merton (Torres Albero \& Lamo de Espinosa, 2003, p. 16). Sus primeras producciones académicas fueron reflexiones en torno al pensamiento de Émile Durkheim y posteriormente se vinculó con las elaboraciones realizadas por Talcott Parsons, a partir de su trabajo fundamental La estructura de la acción social, publicado en 1937.

Entre finales de la década del treinta y principios de la del cuarenta, el proyecto de investigación que coordinaba Lazarsfeld se trasladó a la Universidad de Columbia. Allí fue donde, en 1943, Merton se incorporó al Bureau of Applied Social Research y desarrolló distintos aportes conceptuales a los estudios realizados por Lazarsfeld. Como sostienen Torres Albero y Lamo de Espinosa (2003), de esa primera experiencia en el grupo de investigación surgió su trabajo publicado en 1946, Mass Persuassion, "basado en los estudios sobre las emisiones de radio", en el que se conjugaron el trabajo empírico con la reflexión teórica (p. 19).

\section{El programa académico-intelectual de la Mass Communication Research}

Entre 1939 y 1940, un conjunto de expertos e investigadores se reunieron en Nueva York bajo el auspicio de la Fundación Rockefeller para dialogar sobre la situación de la investigación sobre la comunicación de masas en un contexto que denominaron de 
crisis (Bryson, Free, Gorer, Lasswell, Lazarsfeld, Lynd, Marshall, Siepmann, Slesinger \& Waples, 2001 [1940], p. 188). En ese encuentro participaron Lasswell, Lazarsfeld, Robert S. Lynd, John Marshall y Charles Siepmann, entre otros, y se redactó un memorándum titulado "La investigación sobre la comunicación de masas". El texto se convirtió en una declaración en favor de la investigación de los mass media como la prensa, la radio y el cine (Bryson et al., 2001 [1940], p. 185). Este memorándum presentaba las características de un programa o un plan de trabajo: indicaba la pertinencia de la problemática, pretendía justificar teórica y políticamente la investigación, planteaba objetivos y fines, exponía una serie de estrategias metodológicas, consideraba la fundación de una institución que articulara a los distintos investigadores y proyectos y, por último, planteaba la viabilidad en términos económicos, es decir, los costos de la investigación sobre la comunicación de masas.

No se pretende dar cuenta aquí de todo el programa, sino de una serie de elementos que, consideramos, orientaron las preocupaciones de los investigadores norteamericanos de la primera mitad del siglo XX. Si el punto de partida se encontraba en una sociedad en situación de crisis, esta era percibida como "tiempos difíciles" en los cuales Estados Unidos debía hacer frente a la "necesidad de adaptarse a un mundo en mutación” (Bryson et al., 2001 [1940], p. 186). En este marco, los autores buscaron interpelar al Estado insinuándole que solo se podría salir de la crisis y de los "peligros" -de una eventual guerra ${ }^{-1}$ si se preservaban "los modos de vida que amamos los americanos" mediante la construcción de la aceptación ciudadana (p. 186).

Este proceso de construcción solo sería posible si las políticas públicas se reorientaban a obtener información que diera cuenta de la predisposición y las necesidades del público, y la investigación sobre la comunicación de masas era "una nueva arma segura para alcanzar este fin" (p. 186). Al respecto, se destacó que tenía gran valor movilizar recursos y servicios hacia un fin como el de guiar las ideas y los sentidos de la opinión pública. En esta preocupación política e intelectual acerca de cómo construir consensos se situó la pregunta por las modalidades de incorporación de los medios masivos en los procesos más amplios de organización político-cultural con el fin de construir la unidad nacional (p. 188). En este contexto se inscribía el interrogante, ya clásico, sobre qué hacen los medios en la sociedad.

Complementariamente, entendemos que esta propuesta de los expertos se orientó a pensar distintas estrategias para saber no solamente qué hacen los medios, sino cómo hacer para que estos operen según un proyecto político-cultural específico. En este sentido, se daba un desplazamiento desde la producción de saberes hacia la intervención y la práctica. Tal preocupación, que motorizó el memorándum, fue el eje

Si se desataba un conflicto bélico, podía tener efectos en términos de crisis orgánica, como ya había ocurrido tras el crac de 1929. Para más información, ver Hobsbawm (2006, p. 111). 
Austral Comunicación

Volumen 7 número 2 (diciembre de 2018): 155-172. ISSN 2313-9129

sobre el que se desarrollarían las futuras investigaciones de una franja de estudiosos e intelectuales norteamericanos que debatieron sobre el rol de los medios masivos en la sociedad. De manera particularmente clara se planteaba el programa que ocho años después consagraría a Lasswell (1985 [1948]) en su clásico trabajo, "Estructura y función de la comunicación en la sociedad", es decir, la definición de las cuatro preguntas básicas del estudio de las comunicaciones de masas: "quién dice qué", "en qué canal", "a quién" y "con qué efecto" (Bryson et al., 2001 [1940], pp. 196-197).

De este modo, no solo se delimitaba un campo de problemas, sino que, yendo un poco más allá, el programa pretendía legitimar doblemente al especialista en comunicación: como científico capaz de producir saberes en torno a los medios masivos y como especialista en la formulación de políticas públicas. En concreto, los académicos formados científicamente, desde su saber específico, podían incorporarse en los estamentos del Estado y colaborar con la producción de programas de radio y comunicados de prensa en los que se buscara orientar a la población hacia un objetivo común (Bryson et al., 2001 [1940]). Consideraban, en definitiva, que la situación de crisis volvía necesaria la unidad nacional, que implicaba una alianza y cooperación entre los sectores públicos y privados. En estas circunstancias, asumían que el conocimiento derivado de la investigación iba a ser necesario al momento de articular decisiones respecto a la política pública. En cualquier caso, sería de suma importancia "medir los efectos que tienen o pueden llegar a tener las comunicaciones de masas” (p. 95).

\section{Medios y cultura de masas: \\ entre la democratización y la decadencia cultural}

Si bien el memorándum "La investigación sobre la comunicación de masas" (Bryson et al., 2001 [1940]) planteaba un proyecto general que reunía a distintos investigadores, lejos estamos de suponer que los firmantes realizaron sus trabajos de modo orgánico. Por el contrario, consideramos que, si bien aquel operó como una brújula orientativa, se trazaron distintos caminos y se establecieron diferencias dentro del pensamiento norteamericano acerca de la comunicación.

Atentos al desarrollo político de la sociedad norteamericana, Lazarsfeld y Merton (1985 [1948]), en su artículo "Comunicación de masas, gustos populares y acción social organizada", analizaban cuáles eran las transformaciones que se estaban desarrollando en los Estados Unidos. Con respecto a las medidas tomadas durante el gobierno de Franklin Roosevelt, los investigadores estimaban que ese "movimiento reformista" había transformado la vida de los trabajadores norteamericanos. Se referían concretamente a la reducción de las jornadas laborales de sesenta a cuarenta horas semanales, el descenso progresivo del trabajo infantil y la institucionalización de la enseñanza gratuita (p. 176). Esta reconfiguración de la vida cotidiana de la clase trabajadora abría 
un margen más amplio para el desarrollo de sus prácticas culturales. Por ello, para los investigadores, estas nuevas condiciones habilitadas por las "victorias reformistas" lograban mayor tiempo de ocio y mayor acceso al patrimonio cultural (p. 176).

De todos modos, esta incorporación de las masas en el espacio público y el consumo cultural trajo consigo distintos interrogantes. $\mathrm{Al}$ respecto, una franja de la intelectualidad veía en los medios masivos una amenaza para la cultura. En efecto, un rector de la Universidad de Chicago llegó a afirmar (según cita en Siepmann, 1953, p. 2): "Bajo el impacto de la televisión, me imagino que en Estados Unidos ha de llegar un día en que las gentes no sepan leer ni escribir, ni lleguen a ser superiores a ciertas formas de la vida vegetal". El debate acerca de la "decadencia de la cultura" producida por los medios masivos estaba en boga (Swingewood, 1979).

En sentido contrario a esa lectura, Lazarsfeld y Merton planteaban las transformaciones culturales en términos históricos y sociológicos. Por un lado, entendían que con la ampliación de la audiencia como resultado de la "educación popular y con la aparición de las nuevas técnicas de la comunicación de masas, se había desarrollado un mercado enormemente ampliado para las artes" (Lazarsfeld \& Merton, 1985 [1948], p. 184). Este proceso democratizador, en todo caso, había despojado del monopolio cultural a una limitada elite aristocrática, cuyo gusto estético y cuya exigencia selectiva se habían transformado en la pauta artística dominante (p. 183).

A propósito de lo anterior, nos interesa acentuar dos hipótesis de trabajo que presentaron estos investigadores y que se distinguieron de otras propuestas en el marco en que se daba el debate. Lazarsfeld y Merton insinuaban si acaso no habría ocurrido que los mass media hubiesen "robado" -robbed, en el original- a la elite intelectual y artística sus consumos habituales al producirlos masivamente. Y esta pregunta, consideramos, implicaba repensar las relaciones de poder: ya no verticalmente desde los medios masivos hacia las masas, sino como una presión desde abajo que ejercían las masas sobre los productores culturales. ${ }^{2}$ Entendemos esto ateniéndonos a la inquietud de estos autores: la necesidad de investigar la "presión ejercida por la audiencia masiva sobre los individuos creativos" para amoldarlos a sus propios gustos (p. 184).

En este sentido, señalaban, un tanto irónicamente, que una franja de los intelectuales se sintió estafada -o engañada, para ser más precisos- porque los mass media les arrebataron a los dirigentes culturales "los frutos de sus victorias": en lugar de consumir Shakespeare, Beethoven o quizás Kant, les interesaba el star system de la época: Faith Baldwin, Johnny Mercer o Edgar Guest. Y consideraban que era esa "traición”, así percibida, la que producía esa actitud -distancia y desconfianza- de los intelectuales respecto a los medios masivos (p. 176).

La cuestión de la influencia de los medios masivos, los productores culturales y las masas revivió, en parte, un momento del debate entre Lazarsfeld y Theodor Adorno. Ver Wiggershaus (2011, pp. 298-308). 
Austral Comunicación

Volumen 7 número 2 (diciembre de 2018): 155-172. ISSN 2313-9129

\section{Límites y alcances de las funciones de los medios}

Respecto a las funciones sociales de los medios, cuestión que se muestra central en los estudios norteamericanos, Lasswell no tenía en el horizonte las mismas preocupaciones que Lazarsfeld y Merton. En su artículo "Estructura y función de la comunicación en la sociedad" (1985 [1948]), él pensaba específicamente a los mass media como dimensión organizativa de la actividad política y administrativa. Por ello hacía referencia, en términos de ejemplificación, a las modalidades de intervención del Estado frente a diversas situaciones.

Planteaba como hipótesis explicativa de su propuesta los modos como el Estado actuaba frente a situaciones en las que la "verdad" acerca de tal o cual hecho no era compartida por las capas dirigenciales y la sociedad (Lasswell, 1985 [1948], p. 204). En este marco, sostenía que se pretendía controlar la circulación de la comunicación para reorientar las ideas de la población en beneficio del proyecto de las clases gobernantes y así evitar conflictos sociales (p. 204).

La cuestión de fondo, que creemos que es una dimensión determinante en la propuesta de Lasswell, es el anudamiento entre función y estructura al pensar los medios masivos. La estructura, en este contexto, es el concepto que da inteligibilidad a las funciones. Es decir, es la manera de articular el acto de la comunicación con todo el proceso social (p. 194). De este modo, se comprende que, al ser subsidiarias de la estructura, las funciones y su tipologización no serían siempre las mismas.

Según Lasswell, en la estructura social particular -léase: sociedad norteamericana de la década del cuarenta-, las funciones de la comunicación eran tres: la supervisión o vigilancia del entorno, la articulación coherente de los distintos elementos de la sociedad ante situaciones de crisis y, por último, la tarea de transmitir la herencia social de una generación a la siguiente (p. 205). La propuesta lasswelliana, consideramos, se ajustaba fielmente al memorándum firmado en 1940: una de las preguntas que subyacía en las ideas del investigador podría ser enunciada de la siguiente manera: ¿de qué modo la comunicación puede operar en la construcción de gobernabilidad ante situaciones de crisis orgánica?

A diferencia de lo anterior, Lazarsfeld y Merton pensaron ciertas funciones pero en un plano político y cultural más general. Y además, planteaban limitaciones -cuando no escepticismo- acerca de la incorporación de los medios en los proyectos sociales como producto de su estructura empresarial-comercial. Según sabemos, para ellos los medios cumplían dos funciones: de atribución de status y de aplicación forzosa de normas sociales. A su vez, como disfunción narcotizante, se daba un proceso de sustitución del saber acerca de la política por sobre la participación política (Lazarsfeld \& Merton, 1985 [1948], pp. 177-180). Es decir, se operaba un pasaje desde "la participación activa hacia el conocimiento pasivo" (p. 181). 
Emiliano Sánchez Narvarte

Entre intelectuales y expertos: una aproximación a los debates norteamericanos

sobre los medios de comunicación (1940-1950)

Teniendo en cuenta que son diferentes las funciones atribuidas a los medios en ambas propuestas, queremos acentuar las dificultades que hallaron Lazarsfeld y Merton en la incorporación de los mass media a los programas sociales. Al señalar ciertos problemas, se distanciaban de Lasswell, que daba a entender una articulación entre políticas públicas y medios aparentemente coherente y sin conflicto. Por el contrario, estos autores consideraban que los medios eran empresas privadas y administradas para obtener beneficio $^{3} \mathrm{y}$, al ser financiados por la pauta publicitaria, el contenido quedaba determinado por las grandes firmas comerciales que presionaban sobre las empresas (p. 182). Por ello sostenían que los medios contribuían al mantenimiento del sistema y lo reafirmaban al no facilitar el pensamiento crítico acerca de él. Y, finalmente, advertían que no cabía "confiar en ellos para que elaboren cambios en la estructura [...] [L]os objetivos sociales son abandonados por los medios cuando chocan con los intereses económicos" (p. 182).

De todos modos, intentaron formular una alternativa para la utilización de los medios para objetivos sociales. Propusieron lo que denominaron complementariedad entre la utilización de las redes nacionales de comunicación y las prácticas cotidianas y dialógicas -puntualmente, el face-to-face contact-, fundándose en distintas experiencias en los Estados Unidos, así como también en las prácticas político-comunicacionales en la Unión Soviética. Básicamente, indicaban que, en sí mismos, como ya se dijo, los mass media no motivaban cambios, sino que pretendían el mantenimiento del orden. Por ello, proponían un proceso combinado de propaganda a través de una red de ámbito nacional, la distribución coordinada de periódicos y folletos y "las discusiones cara a cara localmente organizadas por grupos pequeños". Esta tarea combinada facilitaría el intercambio de opiniones y así sería posible persuadir y transformar las prácticas habituales de los grupos sociales (p. 191).

\section{Acerca de la influencia política de los medios: un debate}

Como se vio anteriormente, Gladys y Kurt Lang habían realizado sus primeras elaboraciones en torno a la relación entre los medios y la sociedad, en el marco de los proyectos político-militares del gobierno norteamericano. En definitiva, se trataba de construir estrategias de interpelación a la sociedad mediante el uso de la propaganda política y, posteriormente, medir sus efectos en determinadas políticas públicas y conflictos bélicos.

Por su parte, los resultados de las distintas investigaciones de Lazarsfeld y Merton se orientaban en un sentido opuesto: matizaban tal efecto de los mass media. Según los estudios realizados por estos autores, entre los medios masivos y la sociedad emergía

\footnotetext{
Según Lazarsfeld y Merton (1985 [1948]), esto no era natural ni inherente. Por ello daban cuenta de que en Inglaterra y en Rusia, "la radio está, para todo fin y propósito, controlada y operada por el gobierno, de la que es propiedad" (p. 181).
} 
Austral Comunicación

Volumen 7 número 2 (diciembre de 2018): 155-172. ISSN 2313-9129

una multiplicidad de elementos, dimensiones, disposiciones de los sujetos que opacaba la relación lineal entre medios y audiencia que otros estudios, supuestamente, habían certificado. Veamos algunos ejes de ese debate.

En el ya citado artículo de Lazarsfeld y Merton (1985 [1948]), así como también en las investigaciones previas del mismo Lazarsfeld (1940, 1985 [1953]) y en su trabajo colectivo junto a Berelson y McPhee (1985 [1954]), se ponía en duda el "exagerado" papel atribuido a los mass media (Lazarsfeld \& Merton, 1985 [1948], p. 186). De hecho, juzgaban que la hipótesis del "estímulo hipodérmico en un sujeto inerte" era una formulación ingenua para pensar los efectos políticos de las comunicaciones de masas (Lazarsfeld et al., 1985 [1954], p. 411).

Ya en su trabajo Radio and the printed page (1940), Lazarsfeld observaba -al igual que Wolf (2013, p. 55)- que, en todo caso, la incidencia de la radio y la prensa en las prácticas cotidianas no obedecía a sus características inherentes, sino al marco social legislaciones laborales, transformaciones educativas- en el que estos se desempeñaban (Lazarsfeld, 1940, p. 329). Además, el futuro rol de la radio en la cultura norteamericana iba a depender, ni más ni menos, de las "fuerzas sociales que prevalecieran en un momento determinado" (p. 330). Esta consideración planteaba que la cuestión de la funcionalidad o la utilidad de los mass media se relacionaría con los proyectos político-culturales particulares (p. 331).

La inscripción sociológica de los estudios recién nombrados se orientó a preguntar, esquemáticamente, sobre el entramado social, cultural y político desde el cual la audiencia decodificaba los mensajes de la prensa y la radio. Sobre esto alertaba Merton cuando afirmaba que las investigaciones en sociología de las comunicaciones de masas debían incorporar la pregunta por los nexos de sociabilidad y las redes de relaciones interpersonales (Merton, 1949, p. 193). Sin esta dimensión, no se podrían entender las decisiones o elecciones de las personas.

Lazarsfeld, por su parte, más circunscrito a la interrogación sobre el mensaje político, estimaba que la participación de los ciudadanos en distintas organizaciones acentuaba su predisposición a consumir cierto tipo de información política. Y, en todo caso, siempre el entorno social tamizaba la eficacia de la propaganda (Lazarsfeld, 1985 [1948], pp. 398-399).

En ese entorno social Lazarsfeld, Berelson \& McPhee (1985 [1954]) inscribían la figura del líder de opinión. Este líder -o referente intelectual, diremos nosotros- traducía lo que circulaba por los mass media a través de las redes y los grupos de participación locales. Por ello es que, alejándose de perspectivas más lineales, los autores consideraban que no se podía entender a los sujetos como individuos aislados, porque estaban situados en un sistema de discusión (p. 411). Justamente este sistema se vinculaba con determinados niveles de participación cívica que predisponían a la audiencia a leer y 
escuchar ideas con las que ya estaba de acuerdo (p. 429). Por lo tanto, sostenían, los medios de comunicación, antes que "convertir" ideológicamente al ciudadano, cristalizaban y reforzaban lo que ya pensaba (p. 426).

Pero había algo de todo lo que argumentaban Lazarsfeld y Merton que al matrimonio formado por Gladys y Kurt Lang no lo terminaba de convencer. Más por obstinación que por los datos arrojados de sus propias investigaciones, ellos afirmaban:

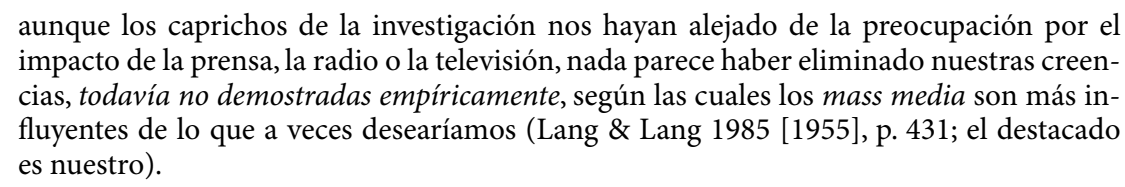

Por eso, discutían las afirmaciones efectuadas por Merton, Lazarsfeld y todo su equipo de trabajo. Aseguraban que las nociones sobre la política, los partidos, los sentidos acerca de los candidatos, no podían "ser satisfactoriamente explicadas a través del estudio de las redes de comunicación locales" (p. 443) porque, en todo caso, lo que allí circulaba y se discutía, seguían siendo ideas "afectadas por lo que los mass media presentan" (p. 443). En ese sentido, pensaban que los medios masivos estructuraban una realidad más amplia que el énfasis local puesto por las otras investigaciones. De algún modo, planteaban que el contenido de los medios "se filtra, aunque la gente no le preste gran atención" y su influencia tiene carácter acumulativo (p. 444).

Se podría agregar que los efectos a largo plazo, para esta posición, se van acumulando y sedimentando a través del tiempo. La cuestión, admitirán los Lang, era que, si bien esta influencia estaba presente y todos estaban expuestos e involucrados en ella, sin embargo, era un problema que escapaba a la labor investigadora (p. 448).

Este escapar a la tarea de investigación incorporaba otro elemento a la discusión, vinculado con la dificultad de corroborar el poder de influencia que tenían los medios. Si bien no profundizaron en este punto, la comprobación del poder de los medios masivos, su capacidad de cambiar actitudes, ideas o significaciones en los ciudadanos, iba a atravesar y a producir grandes debates dentro del campo académico de la comunicación, no solo en Norteamérica, sino también en otras regiones del mundo.

\section{A modo de cierre}

Sin duda, mucho se ha escrito acerca del pensamiento norteamericano sobre la comunicación, desde perspectivas más o menos críticas. Lo cierto es que las reflexiones de Lasswell, Lazarsfeld o Merton, por nombrar solo algunos, son consideradas fundadoras de los estudios en comunicación a nivel internacional.

Luego de indagar los debates de la Mass Communication Research en torno a las funciones y los efectos de los mass media, dos cuestiones deben ser puestas de relieve. 
Austral Comunicación

Volumen 7 número 2 (diciembre de 2018): 155-172. ISSN 2313-9129

La primera de ellas está ligada a la relación entre campos que muchas veces aparecen como radicalmente diferentes, cuando no antagónicos. Pensamos particularmente en los espacios de intervención de profesionales e intelectuales. Cierta clasificación dicotómica ha establecido los rótulos de administrativas o críticas para las investigaciones -las primeras serían las norteamericanas; las segundas, las de la Escuela de Frankfurt (Wolf, 2013)-, que, más que explicar a qué hacen referencia tales estudios, son reduccionismos propios de una modalidad de autoposicionarse dentro de un campo -que merece ser problematizada-, o de una "miopía ideológica", más que de una genealogía del pensamiento acerca de la comunicación (Galindo Cáceres, 2007, p. 6).

Para salir de esas falsas dicotomías y problematizar la producción del pensamiento comunicacional norteamericano, es pertinente no marcar dos figuras, el intelectual y el experto, como puntos extremos de una línea. Por el contrario, propusimos pensar sus posicionamientos y elaboraciones desde la noción de espacios de intersecciones múltiples (Neiburg \& Plotkin, 2004, p. 18). El espacio, en el caso particular norteamericano, se definió por el Estado, la academia, el sector privado y el campo intelectual. Desde este cruce productivo de zonas diferentes, una franja de académicos norteamericanos produjo conocimientos sobre la comunicación y la sociedad.

La categoría de productores culturales de Williams (2015 [1981], p. 178), por su parte, permite pensar a los referentes norteamericanos ya no simplemente como teóricos. Desde una perspectiva más amplia, al inscribir a los intelectuales y expertos en redes de sociabilidad y tramas institucionales más extensas, se sitúa el problema en otras coordenadas, en las que la producción de saberes se halla en un entramado de disputas en el que la exposición de una teoría no debe invisibilizar que surge en procesos de luchas contra otras a las que se opone. En este sentido, siguiendo a Williams (1997 [1989], p. 215), ubicar a los productores de ideas en procesos más amplios permite pensar las obras y las conceptualizaciones emergentes como respuestas específicas a los problemas de la sociedad que tales agentes están experimentando.

Un segundo aspecto que merece reflexión está vinculado con las interpretaciones que se han realizado desde las academias latinoamericanas de comunicación. Galindo Cáceres (2007) plantea que se ha producido históricamente una versión acerca del pensamiento norteamericano -en particular, sobre el funcionalismo- en la que "no importa si sabemos o no qué pasa con el funcionalismo y la comunicación, lo que importa es tener una postura, una visión de descalificación" (p. 1). Esta situación ha llevado a producir interpretaciones que no conducen a la comprensión del pensamiento norteamericano, sino a su desconocimiento (p. 6). Esta propuesta implica repensar las condiciones de recepción de la teoría norteamericana sobre medios masivos en América Latina hacia los sesenta y setenta. Es decir, de qué modo en las interpretaciones de referentes e intelectuales de la comunicación del Cono Sur se han configurado 
críticas más vinculadas con el propio contexto latinoamericano ${ }^{4}$ que con las específicas condiciones de producción de las ideas norteamericanas. En este sentido, siguiendo a Pierre Bourdieu (2011 [1990], p. 161), el hecho de que los textos circulen sin sus contextos, que no se importe el campo de producción de los materiales y estos se inserten en condiciones diferentes, genera importantes malentendidos en cuanto son leídos desde un campo de recepción interpelado por otras inquietudes y problemáticas. Por el contrario, entendemos que solo se comprenden las elaboraciones del pensamiento norteamericano sobre comunicación cuando se las sitúa en las preocupaciones y los dilemas de su época. Al respecto, es necesario empezar a distinguir y diferenciar los matices teóricos dentro de esa tradición intelectual, así como también dar cuenta de los debates en los que emergieron tales perspectivas. Consideramos, en definitiva, que estas operaciones permitirán otorgar mayor inteligibilidad a esos procesos de producción de conocimiento sobre la comunicación y establecer diálogos productivos con las problemáticas y los desafíos sociales en la actualidad.

\section{Referencias}

Bryson, L., Free, L., Gorer, G., Lasswell, H., Lazarsfeld, P., Lynd, R., Marshall, J., Siepmann, C., Slesinger, D. \& Waples, D. (2001 [1940]). La investigación sobre la comunicación de masas. Revista Española de Investigaciones Sociológicas, 95, 185-210.

Bourdieu, P. (2011 [1990]). Las condiciones sociales de la circulación de las ideas. En Intelectuales, política y poder. Buenos Aires: Eudeba.

Chartier, R. (1992). El mundo como representación. Estudios sobre historia cultural. Barcelona: Gedisa.

Cole, J. (2004). Paul F. Lazarsfeld: his scholarly Journey. Discurso pronunciado en An International Symposium in Honor of Paul Lazarsfeld, Bruselas, Bélgica. Obtenido de http://www.columbia.edu/cu/univprof/jcole/_pdf/2004Lazarsfeld.pdf.

De Moragas, M. (ed.) (1985). Sociología de la comunicación de masas. Barcelona: Gustavo Gili.

De Moragas, M. (2011). Interpretar la comunicación. Estudios sobre medios en América y Europa. Barcelona: Gedisa.

Fleming, D. \& Bernard, B. (1969). The intellectual migration: Europe and America, 19301960. Massachusetts: Harvard University.

Galindo Cáceres, L. (2007). La sociología funcionalista y la comunicología histórica. Un apunte historiográfico de una versión histórica. Question, 1(16), 1-7. Obtenido de http://perio.unlp.edu.ar/ojs/index.php/question/article/viewFile/443/367.

\footnotetext{
4 El contexto latinoamericano de recepción de la teoría norteamericana fue trazado por procesos de radicalización política, y rechazo a la política norteamericana y sus modalidades de institucionalización en las academias latinoamericanas.
} 
Austral Comunicación

Volumen 7 número 2 (diciembre de 2018): 155-172. ISSN 2313-9129

Gómez Aguirre, M. \& Resico, M. (2009). La crisis de 1930 y las políticas del New Deal. Un examen desde la Economía y las Instituciones. Ensayos de Política Económica, 3, 27-52. Obtenido de http://bibliotecadigital.uca.edu.ar/repositorio/revistas/ crisis-1930-politicas-new-deal.pdf.

Graham, S. (2015). Culture and Propaganda. The Progressive Origins of American Public Diplomacy, 1936-1953. Farnham: Ashgate.

Hobsbawm, E. (2006 [1994]). Historia del siglo XX. Buenos Aires: Crítica.

Jahoda, M., Lazarsfeld, P. \& Zeisel, H. (2009 [1930]). Marienthal: The Sociography of an Unemployed Community. Nueva Brunswick: Transaction Publisher.

Jay, M. (2017). Exilios permanentes. Ensayos sobre la migración intelectual alemana en Estados Unidos. Buenos Aires: El Cuenco de Plata.

Lang, K. \& Lang, G. (1985 [1955]). Los mass media y las elecciones. En De Moragas, M. (ed.). Sociología de la comunicación de masas (pp. 431-451). Barcelona: Gustavo Gili.

Lasswell, H. (1985 [1948]). Estructura y función de la comunicación en la sociedad. En De Moragas, M. (ed.). Sociología de la comunicación de masas (pp. 192-206). Barcelona: Gustavo Gili.

Lazarsfeld, P. (1940). Radio and the printed page. Nueva York: Duell, Sloan \& Pearce.

Lazarsfeld, P. (1985 [1953]). La campaña electoral ha terminado. En De Moragas, M. (ed.). Sociología de la comunicación de masas (pp. 394-409). Barcelona: Gustavo Gili.

Lazarsfeld, P. \& Merton, R. (1985 [1948]). Comunicación de masas, gustos populares y acción social organizada. En De Moragas, M. (ed.). Sociología de la comunicación de masas (pp. 172-191). Barcelona: Gustavo Gili.

Lazarsfeld, P., Berelson, B. \& McPhee, W. (1985 [1954]). Procesos políticos: la misión de los mass media. En De Moragas, M. (ed.). Sociología de la comunicación de masas (pp. 410-430). Barcelona: Gustavo Gili.

Mattelart, A. (1996). La comunicación-mundo. Historia de las ideas y de las estrategias. México: Siglo XXI.

Mattelart, A. \& Mattelart, M. (1997). Historia de las teorías de la comunicación. Barcelona: Paidós.

McQuail, D. (2003 [1983]). Teoria da comunicação de massas. Lisboa: Fundação Calouste Gulbenkian.

Merton, R. (1949). Patterns of influence: a study of interpersonal influence and of communications behavior in a local community. En Lazarsfeld, P. \& Stanton, F. (comps.). Communications research, 1948 -1949 (pp. 180-222). Nueva York: Harper $\&$ Brothers.

Müller-Doohm, S. (2005). Adorno: A Biography. Cambridge: Polity. 
Neiburg, F. \& Plotkin, M. (2004). Intelectuales yexpertos. La constitución del conocimiento social en la Argentina. Buenos Aires: Paidós.

Siepmann, C. (1953). El siglo de la televisión. El correo de la UNESCO, 6(3), 2-3.

Supa, D. (2009). The Origins of Empirical versus Critical Epistemology in American Communication. American Communication Journal, 11(3), 1-11. Obtenido de http://ac-journal.org/journal/2009/Fall/4TheOriginsofEmpirical.pdf.

Swingewood, A. (1979). El mito de la cultura de masas. México: Premiá Editora.

Torres Albero, C. \& Lamo de Espinosa, E. (2003). In memorian Robert K. Merton (1910-2003). Revista Española de Investigaciones Sociológicas, 100, 13-26.

Wiggershaus, R. (2011 [1986]). La Escuela de Fráncfort. Buenos Aires: Fondo de Cultura Económica.

Williams, R. (1997 [1989]). La política del modernismo. Buenos Aires: Manantial.

Williams, R. (2015 [1981]). Sociología de la cultura. Buenos Aires: Paidós.

Wolf, M. (2013 [1987]). La investigación de la comunicación de masas. Críticas y perspectivas. Buenos Aires: Paidós. 